\title{
Adolescents' Perceptions of Cycling versus Walking to School: Understanding the New Zealand Context
}

\author{
Sandra Mandica, Debbie Hopkins ${ }^{b}$, Enrique García Bengoechea ${ }^{c}$, Charlotte \\ Flahertyd $^{\mathrm{d}}$, John Williams ${ }^{\mathrm{e}}$, Leiana Sloane ${ }^{\mathrm{a}}$, Antoni Moore ${ }^{\dagger}$, John C. Spenceg.
} Accepted for a publication in the Journal of Transport and Health. DOI: 10.1016/j.jth.2016.10.007

aSchool of Physical Education, Sport and Exercise Sciences, University of Otago, Dunedin, New Zealand; sandra.mandic@otago.ac.nz; sloaneleiana@gmail.com

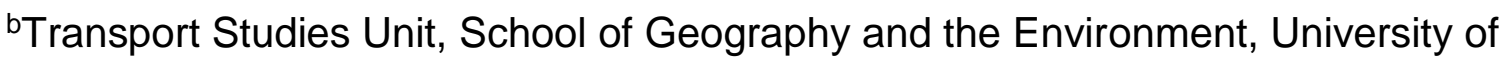
Oxford, United Kingdom; debbie.hopkins@ouce.ox.ac.uk 'Participatory Research at McGill, Department of Family Medicine, McGill University, Montreal, Canada; enrique.garcia@mail.mcgill.ca

dDunedin City Council, Dunedin, New Zealand; charlotte.flaherty@dcc.govt.nz eDepartment of Marketing, University of Otago, Dunedin, New Zealand; john.williams@otago.ac.nz 'School of Surveying, University of Otago, Dunedin, New Zealand; tony.moore@otago.ac.nz 9Faculty of Physical Education and Recreation, University of Alberta, Edmonton, Canada; ic.spence@ualberta.ca

\section{Corresponding author:}

Sandra Mandic, PhD

Senior Lecturer

School of Physical Education, Sport and Exercise Sciences

University of Otago

PO Box 56

Dunedin, New Zealand

Work phone: +64 34795415

Fax: $\quad$ +6434798309

E-mail: $\quad$ sandra.mandic@otago.ac.nz 
Funding: This work was supported by National Heart Foundation of New Zealand (1615), Lottery Health Research Grant (Applic 341129), University of Otago Research Grant (UORG 2015), Dunedin City Council and internal grants from the School of Physical Education, Sport and Exercise Sciences, University of Otago.

\section{Abstract}

Background: Cycling to school is less common than walking in many developed countries. This cross-sectional study compared correlates and perceptions of walking versus cycling to school in Dunedin adolescents living $\leq 4 \mathrm{~km}$ from school.

Methods: Adolescents ( $n=764 ; 44.6 \%$ males; $15.2 \pm 1.4$ years) from 12 secondary schools completed an online survey about perceptions of walking and cycling to school. Distance to school was calculated using Geographic Information Systems network analysis.

Results: Overall, $50.8 \%$ of adolescents walked and $2.1 \%$ cycled to school, $44.1 \%$ liked cycling for recreation and $58.8 \%$ were capable/able/confident to cycle to school. Adolescents expressed more positive experiential (walking: 45.9\%; cycling: $34.9 \%$ ) and instrumental beliefs (walking: 74.2\%; cycling: 59.2\%) towards walking versus cycling to school $(p<0.001)$. Compared to walking, adolescents reported that cycling to school was perceived as less safe by themselves (cycling vs walking; $61.3 \%$ vs $89.8 \%)$ and their parents ( $71.4 \%$ vs $88.6 \%)$ and was less encouraged by their parents (23.0\% vs $67.0 \%)$, peers (18.8\% vs $48.4 \%$ ) and schools (19.5\% vs $30.8 \%$ ) (all $p<0.001$ ). The route to school had fewer cycle paths compared to footpaths ( $37.2 \%$ vs $91.0 \% ; p<0.001)$. Cycle friendly uniforms $(41.4 \%)$, safer bicycle storage at school $(40.1 \%)$, slower traffic $(36.4 \%)$, bus bicycle racks $(26.2 \%)$ and bicycle ownership (32.7\%) would encourage cycling to school. 
Conclusions: Compared to walking, cycling to school among Dunedin adolescents was less common, perceived as less safe and had less social and infrastructure support. Future interventions should focus on creating supportive physical and social environments, and improving road safety for cyclists in New Zealand.

Keywords: Adolescent; physical activity; active transportation; walking; cycling; theory of planned behaviour 


\section{Highlights}

- Low rates of cycling to school in New Zealand adolescents may be contextspecific.

- Compared to walking, cycling to school was less common and perceived as less safe.

- Cycling also received less social and infrastructure support.

- More supportive physical and social environments are needed for promoting cycling. 


\section{Introduction}

Public health goals of increased physical activity and societal interest in alternatives to automobile transportation place a focus on active modes of transport such as walking and cycling. In addition, the need to transition away from motorised transport, particularly the private car, has been publicized as an important way to reduce transport-related greenhouse gas emissions.(1) Transport mode choice is one of the most environmentally-significant decisions made by individuals,(2) and addressing high-carbon personal transport represents a key opportunity to mitigate climate change.

Though walking is a popular form of active transport to school, cycling to school is less common among adolescents in many developed countries including United States,(3) Canada,(4) Spain,(5) Ireland,(6) Australia(7) and New Zealand.(8) In contrast, countries with long cycling traditions, comprehensive cycling-friendly infrastructure and flat landscape such as Belgium(9) and Denmark(10) have higher rates of cycling versus walking to school among adolescents. In New Zealand, rates of walking to school in adolescents have remained relatively stable (26\% in $1989 / 1990 ; 28 \%$ in $2010-2014$ ) while the rates of cycling to secondary school have declined from $19 \%$ in $1989 / 1990$ to $3 \%$ in $2010-2014 .(11)$

Correlates of active transport to school in children and adolescents include demographic characteristics, individual and family factors, school factors, and social and physical environmental factors.(12-15) Most previous studies either examined perceived barriers to walking and cycling to school together or focused on walking.(16) Walking and cycling are different behaviours with distinct characteristics(17) and therefore correlates of those behaviours are also likely to differ.(16, 18) Compared to walking, cycling is faster, covers greater distances, requires more physical skills, demands more specific built environment 
characteristics, and has more prominent traffic safety concerns.(17) In addition, built environment characteristics that predict walking to school in children may not predict cycling.(18) For children, cycling to school is positively associated with high levels of independent mobility,(19) preference to cycle,(20) parental confidence in their child's cycling skills,(19, 20) peer and parental support,(19) and neighbourhood traffic safety,(19) and negatively associated with perceived convenience of driving children to school.(20) Low rates of cycling to school reported in New Zealand adolescents $(8,11)$ may be context-specific and related to the local cycling culture, social norms, non-supportive physical environments and the weather. The city of Dunedin (population: 130,000) is located on the South Island of New Zealand, has a maritime climate with cool and wet weather and diverse topographical landscape with the city centre being surrounded by hills. Coupled with New Zealand's high rates of bicycle-related accidents in adolescents,(21) nation-wide high rates of private vehicle ownership per capita(22), school uniform requirements and lack of school enrolment schemes in Dunedin, these characteristics represent a challenge for promoting active transport to school, and particularly cycling, in this city. Previous studies in adolescents highlighted the importance of social support for active transport(7, 23) and specifically for cycling(19) to school, with a distance of up to $4 \mathrm{~km}$ (2.5 miles) being reasonable for adolescents' cycling to school.(24) Therefore, this cross-sectional study compared perceptions of walking versus cycling to school drawing from the theory of planned behaviour and additional individual, environmental and safety factors among adolescents from Dunedin who lived within $4 \mathrm{~km}$ from school.

\section{Materials and Methods}

\subsection{Participants}


Between February 2014 and April 2015, 1,780 adolescents (13 to 18 years of age) from all 12 secondary schools in Dunedin, New Zealand, participated in the Built Environment and Active Transport to School (BEATS) Study. $(25,26)$ Participants with invalid surveys $(n=38)$, incomplete student consents $(n=20)$, lacking parental consent $(n=59)$, missing survey data $(n=48)$, boarders $(n=162)$, missing distance to school data $(n=11)$, and living $>4 \mathrm{~km}$ from school $(n=668)$ were excluded from the analysis, resulting in a final usable sample size of 774 adolescents.

\subsection{Procedures}

Recruitment procedures have been described in detail elsewhere.(26) Briefly, adolescents were recruited through schools and completed the online survey during class time under researcher assistants' supervision. All adolescents signed consent for taking part in the study. For those under 16 years of age, parents signed either parental opt-out or parental opt-in consent based on the school's preference. The study was approved by the University of Otago Ethics Committee.

\subsection{Assessments}

Questionnaire. The survey included questions about sociodemographic characteristics (age, gender, ethnicity, school, home address, home resources) travel to school habits, perceptions of walking and cycling to school and enablers of cycling to school. Response validity was strengthened by the fact that research assistants were present as participants completed the survey and were monitoring the manner in which participants responded. As a result, 38 invalid surveys were removed from the final sample.

Sociodemographic characteristics. Age was calculated from date of birth at the time of the survey. Home address data were used to determine New Zealand Index of Deprivation (a neighbourhood area deprivation score) as a surrogate for students' socioeconomic status.(27) The deprivation index was recoded from the original 10- 
point scale (1=least deprived to $10=$ most deprived) into five categories: lowest (1-2), middle-low (3-4), middle (5-6), middle-high (7-8) and highest (9-10) deprivation score. Adolescents also reported the number of bicycles and vehicles at home and whether they had a waterproof raincoat. Distance from home to school was determined using the Geographic Information Systems network analysis, based on geocoded addresses.(26)

Travel to school habits. Transport to school ("How do you usually travel to school?') was assessed for different transport modes with response categories "never", "rarely", "sometimes", "most of the time" and "all of the time". Dominant modes of transport to school (used "most/all of the time") and multi-modal transport were used to classify adolescents into active transport (walking, cycling, or riding a non-motorised scooter "most/all of the time"), motorised transport or combined motorised and active transport. Adolescents were also asked how often they walked and cycled to school in the previous two weeks ("never", "almost never", "sometimes", "almost every day" or "every day") and to estimate how long would their journey to school take if they walked or cycled to school ("1-5 minutes", "6-10 minutes”, 11-20 minutes", "21-30 minutes", "31+ minutes” or "I don't know”).

Beliefs about walking and cycling to school. Beliefs related to perceptions of walking and cycling to school were informed by the theory of planned behaviour.(28) Specifically, questions about attitudes towards walking and cycling, subjective norm, perceived behavioural control (PBC) and behavioural intentions were measured with standard items and had good internal reliability.(29) Additional items were developed specifically for this study. Survey items have been described in detail elsewhere.(30)

Briefly, items assessing attitudes towards walking and cycling to school used the stem "For me, regularly walking to school / riding to school by bicycle would be..." 
rated on a six bipolar $(-3$ to +3$)$ semantic differential scales, anchored by the adjectives "dull”-“interesting", “unpleasant”-“pleasant”, "boring"-“stimulating” for experiential beliefs and "unhealthy"-"healthy", "bad"-"good" and "useless"-“useful” for instrumental beliefs.(29) Given the conceptual similarities, individual items for experiential and instrumental beliefs were summed and then averaged to create two composite scores (experiential beliefs composite score: Cronbach's $\alpha=0.87$ (walking), 0.95 (cycling); instrumental beliefs composite score: Cronbach's $\alpha=0.82$ (walking), 0.84 (cycling)).

Subjective norm was assessed using the items "My parents/guardians think I... should/should not walk / ride a bicycle to school", "My friends think I... should/should not walk / ride a bicycle to school" and "One or both of my parents/guardians walk/bicycle frequently". (30) Adolescents also reported frequency of walking/cycling with parents and peers and schools' encouragement for walking/cycling to school.(30)

The capability component of $P B C$ for walking and cycling was measured using an item "How confident are you that you could walk/cycle to school?" (not very confident-very confident).(29) Two additional items were used to assess capability for cycling: "To what extent do you see yourself as being capable of riding a bicycle to school? (incapable-capable)"; and "I believe that I have the ability to ride a bicycle to school" (definitely do not-definitely do).(29) Given the conceptual similarities, the scores for ability/capability/confidence items for cycling to school were summed and then averaged to create a cycling capability composite score (Cronbach's $\alpha=0.90$ ). The autonomy component of PBC to walk/cycle to school was measured using an item "How much personal control do you yourself have over whether or not you walk/ride a bicycle to school?" (no control-complete control). 
Behavioural intentions were measured using two items: "How often do you intend to walk/ride a bicycle to school? (never-frequently), and "I want to regularly walk/ride a bicycle to school (definitely do not-definitely do).(29)

Incentives and personal barriers for walking and cycling to school. Incentives (getting exercise, opportunity to socialize) and personal barriers (time constraints, need for planning, after-school schedule, too much to carry, sweating, feeling tired and lack of interest/desire to walk/cycle to school) were assessed using separate items for each mode of transport to school using a 4-point Likert scale anchored in "strongly disagree" (1) and "strongly agree" (4). Personal barriers were also combined into a composite score (Cronbach's $\alpha=0.85$, and 0.84 for walking and cycling, respectively; all corrected item-total correlations greater or equal than 0.53 ). Environmental barriers and safety. Environmental barriers (distance, lack of footpaths/bike lanes, and weather) and safety perceptions (adolescents' and parental perceptions) related to walking and cycling to school were assessed using separate items for each mode of transport to school. All items were assessed using a 4-point Likert scale anchored in "strongly disagree" (1) and "strongly agree" (4). Route to school. Route to school (hills, insufficient lighting, too much traffic, dangerous crossing(s), boring route) and convenience of being driven to school were assessed for walking and cycling together using a 4-point Likert scale with response options ranging from "strongly disagree" (1) to "strongly agree" (4).

Enablers of cycling to school. Factors that would encourage cycling to school were examined using a statement "I would cycle to school more often if..." focusing on speed of traffic ("if the traffic on the road(s) was slower"), availability of bus bike racks ("if buses had bike racks free of charge"), safer bike storage at school ("if there were safer places to lock up my bike at school"), lockers for storing belongings at school ("if I had a locker at school for storing my things"), cycle friendly uniform ("if I 
had a cycle-friendly uniform"), cycling without a helmet ("if I was allowed to cycle without a helmet") and bike ownership ("if I owned a bike"). These items were assessed using a 4-point Likert scale anchored in "strongly disagree" (1) and "strongly agree" (4).

\subsection{Data Analysis}

Variables assessing perceptions of walking versus cycling using 4-point or 7-point Likert scales were analysed as continuous variables using paired t-tests. For those items, data are reported as both mean \pm SD and frequency (\%) of adolescents agreeing with each statement. To calculate the proportion of adolescents agreeing with each statement, 4-point Likert scale items were also recoded into "disagree" and "agree" and 7-point Likert scale items were recoded as "disagree", "neutral" or “agree”. Data analysis was performed using SPSS Statistical Package (Version 22). To account for multiple tests, a p-value of $<0.001$ was chosen to indicate statistical significance.

\section{Results}

\subsection{Sociodemographic Characteristics}

Among 764 adolescents surveyed (age: $15.2 \pm 1.4$ years; $44.6 \%$ males), $71.8 \%$ had at least one bicycle available for their use and $61.0 \%$ had two or more vehicles at home (Table 1). Average distance to school was $1.9 \pm 1.0 \mathrm{~km}$ (Table 1). Adolescents' estimates revealed that $51.7 \%$ could walk and $58.8 \%$ could cycle to school within 20 minutes and $29.3 \%$ did not know the estimated time to cycle to school (Table 2).

\subsection{Transport to School Habits}

Common modes of transport to school were being driven in a car and walking, whereas only $2.1 \%$ cycled to school (Table 1). Overall, $44.0 \%$ of adolescents used active transport, $42.4 \%$ motorised transport and $13.6 \%$ combined motorised and active transport. The rates of active transport to school did not differ by gender 
(males: 45.0\%, females: 43.1\%; $p=0.050)$, age $(42.5 \%, 41.8 \%, 53.1 \%, 42.1 \%$, $40.4 \%, 38.5 \%$ for age 13 to 18 years, respectively; $p=.129$ ), ethnicity (New Zealand European: $43.6 \%$, Māori: $45.1 \%$, other: $44.9 \%$; $p=.142$ ) or socioeconomic status (41.3\%, 43.2\%, 42.1\%, 49.7\% and $45.7 \%$ for quintiles of neighbourhood deprivation scores from quintile 1 (least deprived) to quintile 5 (most deprived), respectively; $\mathrm{p}=.389)$. In the previous two weeks, $48.7 \%$ of adolescents walked and $1.9 \%$ cycled to school on most days (Table 2). The rates of active transport to school ranged from $19.2 \%$ to $59.1 \%$ per school (Figure 1 ).

\subsection{Route to School}

Approximately one third of adolescents reported too much traffic $(36.0 \%)$, dangerous crossing(s) (32.9\%), too many hills (33.4\%) and boring route $(33.0 \%)$ for walking or cycling to school. Poor lighting (12.2\%) and stray dogs (6.9\%) were less common. More than half of adolescents (52.9\%) found being driven to school convenient.

\subsection{Attitudes, Social Norm, Perceived Behavioural Control and Behavioural Intentions}

On average, adolescents had more positive experiential and instrumental beliefs towards walking compared to cycling to school (Table 3). One third of adolescents perceived that cycling to school was not cool (Table 3). Adolescents received significantly less parental, peer and school support and less parental role modelling for cycling versus walking to school (Table 3). In addition, $16.3 \%$ and $21.0 \%$ of adolescents often cycled with friends or parents, respectively. Intention and selfperceived confidence was lower for cycling compared to walking to school (Table 3).

Using a composite score for cycling capability, $58.8 \%$ of adolescents had high, $10.7 \%$ average and $30.5 \%$ low self-perceived capability to cycle to school.

\subsection{Incentives and Personal Barriers}


Compared to walking, cycling to school provided less opportunity for socializing with friends (Table 4). Although cycling would be faster than walking, cycling to school had more personal barriers (such as too much to carry, after-school schedule, need for planning and getting sweaty) (Table 4). Half of adolescents felt too tired or could not be bothered to walk or cycle to school (Table 4).

\subsection{Environmental Barriers and Safety Perceptions}

One quarter of adolescents living within $4 \mathrm{~km}$ from school perceived that it was too far to walk or cycle to school (Table 4) with perceived distance being a greater barrier for walking versus cycling. Routes to school had less bike lanes compared to walking paths (Table 4). Adolescents reported that both they and their parents considered cycling to school less safe than walking. Wet and cold weather was a major barrier for both modes of transport.

\subsection{Enablers of Cycling to School}

Adolescents perceived that cycle friendly school uniforms (41.4\%), safer bike storage facilities at school (40.1\%), slower traffic on the roads (36.4\%), bus bike racks available free of charge (26.2\%) and bike ownership (32.7\%) would encourage them to cycle to school more often. Having a locker at school for storing belongings (23.6\%) and being allowed to cycle without helmet (21.7\%) were also seen as favourable conditions.

\section{Discussion}

Key findings of the present study include: 1) although half of adolescents enjoyed cycling for recreation, few cycled to school; 2) compared to walking, cycling to school among Dunedin adolescents was less common and a less preferred mode of transport, had more personal barriers, received less social and infrastructure support and was perceived as less safe; 3) approximately one third of adolescents living within $4 \mathrm{~km}$ from school perceived themselves as incapable of cycling to school; 4) 
revisiting school uniform policy, availability of bike racks on the school grounds, reducing traffic speed and availability of a low-cost bike library may increase the rates of cycling to school in adolescents. These findings suggest that social norms and local cycling culture in Dunedin may in part explain the lower preference for cycling versus walking to school in adolescents. Future active transport initiatives should be context-specific and consider the local cycling culture and environment as well as different incentives and personal barriers for cycling versus walking to school. Though nearly half of adolescents living $\leq 4 \mathrm{~km}$ from school walked to school, cycling to school was less common, less preferred and less desirable mode of transport to school among adolescents in the present study. Lower rates of cycling versus walking to school have been observed among adolescents in many developed countries $(3-8,24)$ with a steep decline in cycling to school reported among New Zealand adolescents in the last three decades.(11) In contrast, countries with strong cycling traditions, extensive cycling-friendly infrastructure and flat landscape, consistently report higher rates of cycling versus walking to school (e.g., Denmark(10) and Belgium(9)).

The local cycling culture in Dunedin (New Zealand) may in part explain the lower preference for cycling versus walking to school among adolescents. In the present study, half of adolescents enjoyed cycling for recreation but less than one fifth often cycled with friends or parents and less than a quarter received encouragement from parents, peers and school to cycle to school. In addition, one third of adolescents perceived that cycling to school was not 'cool'. Previous studies highlighted the importance of social support for active transport $(7,23,31,32)$ and specifically for cycling(19) to school in adolescents.

Parental perceptions of personal safety and the dangers posed by traffic (speed and volume) and dangerous intersections have been identified as key parental barriers to 
walking and cycling to school,(33) especially in adolescent girls. $(34,35)$ In the present study, road safety concerns (too much traffic and/or dangerous crossing[s]) on the way to school were reported by one third of adolescents and reducing traffic speed was seen as a favourable strategy for promoting cycling to school. Adolescents also reported that cycling to school was perceived as less safe by themselves and their parents compared to walking. Focus groups with students and parents conducted as a part of the BEATS Study identified a complex range of factors that contribute to perceptions of cycling safety, including features and perceptions of the built environment, traffic safety (including behaviours of other road users), previous cycling experiences (including accidents) and adolescents' cycling skills and on-road experiences (unpublished findings). Taken together, these findings suggest that low perceived social support for cycling to school among Dunedin adolescents may be in part mediated by adolescents' and parental road safety concerns.

Safety concerns including poor walking and cycling facilities, traffic danger and personal safety concerns decrease the likelihood of active transport to school in children and adolescents. $(20,36)$ Specific to cycling, parental confidence in their child's ability to cycle to school mediates the association between the perceived safety and cycling in children.(20) In the present study, adolescents reported that they and their parents perceived cycling to school as less safe compared to walking. In addition, one third of adolescents living $<4 \mathrm{~km}$ from school did not perceive themselves as capable to cycle to school. These findings emphasise the need for not only creating safer environments but also offering programmes such as cycle skills training(30) to improve adolescents' cycle skills.

Other barriers to active transport to school such as the weather, $(37,38)$ hills, $(39)$ high rates of private vehicle ownership $(8,40)$ and convenience of being driven (e.g. 
trip chaining $)(18,37)$ may in part explain the low preference for cycling versus walking to school among Dunedin adolescents. Although half of adolescents in the present study reported cold and wet weather as a barrier to active transport, the weather was not a greater barrier for cycling versus walking to school. One third of surveyed adolescent reported too many hills on the way to school and one-half reported convenience of being driven to school by someone on the way to something else. Considering greater personal barriers to cycling versus walking to school observed in the present study (too much to carry, after-school schedule, need for planning and sweating), future studies should examine if perceived convenience of being driven to school has a greater impact on cycling versus walking to school in adolescents.

School's support for active transport plays an important role in promoting active transport to school $(37,41)$ through activities such as safety education, special events and infrastructure improvements.(41) Our findings suggest that cyclingspecific interventions at the school level should address school uniform policy (e.g., allow students to cycle to school without a school uniform) as well as school grounds infrastructure to provide safe bicycle storage facilities and lockers at school. In the present study, owning a bicycle and having bus bike racks available free of charge were perceived as enablers of cycling to school by one third and one quarter of adolescents, respectively. Therefore, community-based or city-wide interventions such as availability of the low-cost bike library or bus bike racks free of charge may be effective in promoting cycling to school in adolescents. A law requiring the wearing of a bike helmet was a barrier to cycling to school in only one in five adolescents. Other previously suggested interventions $(37,42)$ should be considered for promoting cycling to school such as a minimum busing distance laws, developing a well-connected network of cycle lanes along low traffic streets and encouraging 
both adolescents and their parents to reconsider what constitutes a reasonable cycling distance.

Limitations. This study has limitations that should be recognized. First, the crosssectional design prevents claims about causality. Second, the unique setting of Dunedin may limit generalizability of findings to other cities and countries with different topography, built environment features and cycling culture. Finally, travel patterns from school to home and objective measures of the environment were not available. Despite the limitations, the strengths include a comprehensive survey of factors affecting walking and cycling behaviour in adolescents and a large representative sample of adolescents from one city in New Zealand, with high participation rates among the schools. Future studies should examine walking and cycling as distinct active transport behaviours in different populations and geographic settings.

\section{Conclusions}

Compared to walking, cycling to school was less common in adolescents, was perceived as less safe and had less social and infrastructure support in Dunedin (New Zealand). Future interventions to promote cycling to school should focus on modifying psychosocial barriers such as raising parental awareness of the benefits of cycling to school and increasing parental and peer support for cycling to school. Initiatives designed for promoting cycling in New Zealand adolescents should be comprehensive and focus on building a positive cycling culture, creating supportive physical and social environments, improving road safety and improving adolescents' cycling skills.

\section{Acknowledgements}


The BEATS Study is a collaboration between Dunedin Secondary Schools' Partnership, Dunedin City Council and University of Otago. We would like to acknowledge our research team members, the members of the BEATS Study Advisory Board (Mr Andrew Lonie, Mrs Ruth Zeinert, Dr Tara Duncan, Dr Susan Sandretto, Dr Janet Stephenson), research personnel (research assistants, students and volunteers), and all participating schools and students.

\section{Authors' Contributions}

SM is the principal investigator who conceptualized the overall study, led the project implementation and drafted this manuscript. SM, DH, JW, AM, EGB and JC contributed to the design of the study and questionnaires. SM, JW, AM, EGB and JC obtained research funding. SM, JW, DH, AM and CF conceptualized this particular study. SM, JW and LS were responsible for statistical analysis. AM was responsible for Geographic Information Systems data analysis. All authors have read and approved the final version of the manuscript, and agree with the order of presentation of the authors.

\section{Competing Interests}

Mrs Charlotte Flaherty works at the Dunedin City Council and has been a part of the BEATS Study research team from the study inception. Other authors do not have any competing financial interests.

\section{Funding}

This work was supported by National Heart Foundation of New Zealand [1615], Lottery Health Research Grant [Applic 341129], University of Otago Research Grant [UORG 2015], Dunedin City Council and internal grants from the School of Physical Education, Sport and Exercise Sciences, University of Otago. 


\section{References}

1. Sims R, Schaeffer R, Creutzig F, Cruz-Núñez X, D’Agostom M, Dimitriu D, et al. Transport. Climate Change 2014: Mitigation of Climate Change Contribution of Working Group III to the Fifth Assessment Report of the Intergovernmental Panel on Climate Change. Cambridge, United Kingdom and New York, NY, USA: 2014.

2. Collins $\mathrm{CM}$, Chambers SM. Psychological and situational influences on commuter-transport-mode choice. Environ Behav. 2005;35:640-61.

3. McDonald NC. Active transportation to school: trends among U.S. schoolchildren, 1969-2001. Am J Prev Med. 2007;32(6):509-16.

4. Larsen K, Gilliland J, Hess P, Tucker P, Irwin J, He M. The influence of the physical environment and sociodemographic characteristics on children's mode of travel to and from school. Am J Public Health. 2009;99(3):520-6.

5. Chillon P, Ortega FB, Ruiz JR, Perez IJ, Martin-Matillas M, Valtuena J, et al. Socio-economic factors and active commuting to school in urban Spanish adolescents: the AVENA study. Eur J Public Health. 2009;19(5):470-6.

6. Murtagh EM, Dempster $\mathrm{M}$, Murphy $\mathrm{MH}$. Determinants of uptake and maintenance of active commuting to school. Health Place. 2016;40:9-14.

7. Leslie E, Kremer P, Toumbourou JW, Williams JW. Gender differences in personal, social and environmental influences on active travel to and from school for Australian adolescents. J Sci Med Sport. 2010;13(6):597-601.

8. Mandic S, Leon de la Barra S, Garcia Bengoechea E, Stevens E, Flaherty C, Moore A, et al. Personal, social and environmental correlates of active transport to school among adolescents in Otago, New Zealand. J Sci Med Sport. $2015 ; 18(4): 432-7$. 
9. Van Dyck D, De Bourdeaudhuij I, Cardon G, Deforche B. Criterion distances and correlates of active transportation to school in Belgian older adolescents. Int $\mathrm{J}$ Behav Nutr Phys Act. 2010;7:87.

10. Cooper AR, Wedderkopp N, Wang H, Andersen LB, Froberg K, Page AS. Active travel to school and cardiovascular fitness in Danish children and adolescents. Med Sci Sports Exerc. 2006;38(10):1724-31.

11. Ministry of Transport. 25 years of New Zealand travel: New Zealand household travel 1989-2014. Wellington: Ministry of Transport, 2015.

12. Davison KK, Werder JL, Lawson CT. Children's active commuting to school: current knowledge and future directions. Prev Chron Dis. 2008;5(3):A100.

13. Panter JR, Jones AP, van Sluijs EM. Environmental determinants of active travel in youth: a review and framework for future research. Int $\mathrm{J}$ Behav Nutr Phys Act. 2008;5:34.

14. Pont K, Ziviani J, Wadley D, Bennett S, Abbott R. Environmental correlates of children's active transportation: a systematic literature review. Health Place. 2009;15(3):827-40.

15. Wong BY, Faulkner G, Buliung R. GIS measured environmental correlates of active school transport: a systematic review of 14 studies. Int J Behav Nutr Phys Act. 2011;8:39.

16. Lu W, McKyer EL, Lee C, Goodson P, Ory MG, Wang S. Perceived barriers to children's active commuting to school: a systematic review of empirical, methodological and theoretical evidence. Int $\mathrm{J}$ Behav Nutr Phys Act. 2014;11:140.

17. Krizek KJ, Forsyth A, Baum L. Walking and cycling international literature review. Final report. Victoria, Australia: Department of Transport, 2009. 
18. Schlossberg M, Greene J, Paulsen Phillips P, Johnson B, Parker B. School trips: Effects of urban form and distance on travel mode. J Am Plann Assoc. $2006 ; 72(3): 337-46$.

19. Ducheyne F, De Bourdeaudhuij I, Spittaels H, Cardon G. Individual, social and physical environmental correlates of 'never' and 'always' cycling to school among 10 to 12 year old children living within a $3.0 \mathrm{~km}$ distance from school. Int J Behav Nutr Phys Act. 2012;9:142.

20. Trapp GS, Giles-Corti B, Christian HE, Bulsara M, Timperio AF, McCormack GR, et al. On your bike! a cross-sectional study of the individual, social and environmental correlates of cycling to school. Int $\mathrm{J}$ Behav Nutr Phys Act. $2011 ; 8: 123$.

21. Tin Tin S, Woodward A, Ameratunga S. Injuries to pedal cyclists on New Zealand roads, 1988-2007. BMC Public Health. 2010;10:655.

22. The World Bank. Motor vehicles (per 1,000 people) 2014 [Available from: http://data.worldbank.org/indicator/IS.VEH.NVEH.P3.

23. Carver A, Timperio A, Hesketh K, Crawford D. Are children and adolescents less active if parents restrict their physical activity and active transport due to perceived risk? Soc Sci Med. 2010;70(11):1799-805.

24. Nelson NM, Foley E, O'Gorman DJ, Moyna NM, Woods CB. Active commuting to school: How far is too far? Int J Behav Nutr Phys Act. 2008;5(1):1-9.

25. Mandic S, Mountfort A, Hopkins D, Flaherty C, Williams J, Brook E, et al. Built Environment and Active Transport to School (BEATS) Study: Multidisciplinary and multi-sector collaboration for physical activity promotion. Retos. $2015 ; 28: 197-202$. 
26. Mandic S, Williams J, Moore A, Hopkins D, Flaherty C, Wilson G, et al. Built Environment and Active Transport to School (BEATS) Study: Protocol for a cross-sectional study. BMJ Open. 2016;6:e011196.

27. Salmond C, Crampton P, King P, Waldegrave C. NZiDep: a New Zealand index of socioeconomic deprivation for individuals. Soc Sci Med. 2006;62(6):1474-85.

28. Ajzen I. The theory of planned behaviour. Organ Behav Hum Decis Process. $1991 ; 50(2): 179-2011$.

29. Armitage CJ. Can the theory of planned behaviour predict the maintenance of physical activity? Health Psychol. 2005;24(3):235-45.

30. Mandic S, Flaherty C, Pocock T, Mintoft-Jones A, Frater J, Chillon P, et al. Attitudes Towards Cycle Skills Training in New Zealand Adolescents. Trans Res Part F: Psychol Behav. 2016;42:217-226

31. Simons D, Clarys P, De Bourdeaudhuij I, de Geus B, Vandelanotte C, Deforche B. Factors influencing mode of transport in older adolescents: a qualitative study. BMC Public Health. 2013;13:323.

32. Verhoeven $H$, Simons D, Van Dyck D, Van Cauwenberg J, Clarys P, De Bourdeaudhuij I, et al. Psychosocial and Environmental Correlates of Walking, Cycling, Public Transport and Passive Transport to Various Destinations in Flemish Older Adolescents. PLoS One. 2016;11(1):e0147128.

33. Broberg A, Sarjala S. School travel mode choice and the characteristics of the urban built environment: The case of Helsinki, Finland. Transport Policy. 2015;37:1-10.

34. Esteban-Cornejo I, Carlson JA, Conway TL, Cain KL, Saelens BE, Frank LD, et al. Parental and Adolescent Perceptions of Neighborhood Safety Related to Adolescents' Physical Activity in Their Neighborhood. Res Q Exerc Sport. 2016:1-9. 
35. Carver A, Salmon J, Campbell K, Baur L, Garnett S, Crawford D. How do perceptions of local neighborhood relate to adolescents' walking and cycling? Am J Health Promot. 2005;20(2):139-47.

36. Kerr J, Rosenberg D, Sallis JF, Saelens BE, Frank LD, Conway TL. Active commuting to school: Associations with environment and parental concerns. Med Sci Sports Exerc. 2006;38(4):787-94.

37. Gustat J, Richards K, Rice J, Andersen L, Parker-Karst K, Cole S. Youth walking and biking rates vary by environments around 5 Louisiana schools. J Sch Health. 2015;85(1):36-42.

38. Aibar A, Bois J, Generelo E, García Bengoechea E, Paillard T, Zaragoza J. Effect of weather, school transport, and perceived neighborhood characteristics on moderate to vigorous physical activity levels of adolescents from two European cities. Environ Behav. 2015;47(4):395-417.

39. Timperio A, Ball K, Salmon J, Roberts R, Giles-Corti B, Simmons D, et al. Personal, family, social, and environmental correlates of active commuting to school. Am J Prev Med. 2006;30(1):45-51.

40. McDonald NC. Critical factors for active transportation to school among lowincome and minority students. Evidence from the 2001 National Household Travel Survey. Am J Prev Med. 2008;34(4):341-4.

41. Buliung R, Faulkner G, Beesley T, Kennedy J. School travel planning: mobilizing school and community resources to encourage active school transportation. The J Sch Health. 2011;81(11):704-12.

42. Larouche R, Barnes J, Tremblay MS. Too far to walk or bike? Can J Public Health. 2013;104(7):e487-9. 
Table 1. Sociodemographic characteristics and travel to school habits

\begin{tabular}{|c|c|}
\hline & $\begin{array}{c}\text { Study sample } \\
\qquad n=764\end{array}$ \\
\hline Age (years) & $15.2 \pm 1.4$ \\
\hline \multicolumn{2}{|l|}{ Gender $[n(\%)]$} \\
\hline Males & 341 (44.6) \\
\hline Females & $423(55.4)$ \\
\hline \multicolumn{2}{|l|}{ School year $[\mathrm{n}(\%)]$} \\
\hline Year 9 & 258 (33.8) \\
\hline Year 10 & $162(21.2)$ \\
\hline Year 11 & $141(18.5)$ \\
\hline Year 12 & $107(14.0)$ \\
\hline Year 13 & $96(12.6)$ \\
\hline \multicolumn{2}{|l|}{ Ethnicity [n(\%)] } \\
\hline New Zealand European & $556(72.8)$ \\
\hline Māori & $71(9.3)$ \\
\hline Other & $137(17.9)$ \\
\hline \multicolumn{2}{|c|}{ Neighbourhood deprivation score $[\mathrm{n}(\%)]$} \\
\hline 1 (least deprived) & $184(24.3)$ \\
\hline 2 & $162(21.4)$ \\
\hline 3 & $173(22.9)$ \\
\hline 4 & $143(18.9)$ \\
\hline 5 (most deprived) & $94(12.4)$ \\
\hline Distance to school $(\mathrm{km})$ & $1.9 \pm 1.0$ \\
\hline \multicolumn{2}{|c|}{ Usual transport modes to school [n(\%)] } \\
\hline By car (driven by others) & $292(38.2)$ \\
\hline
\end{tabular}


By car (driving myself)

$28(3.7)$

By school bus

$24(3.1)$

By public transport

$21(2.7)$

On foot

$388(50.8)$

By bike

$16(2.1)$

Other

$18(2.4)$

Active and motorised transport to school [n(\%)]

Active transport

$334(43.3)$

Motorised transport

$335(43.4)$

Combination of active and motorised transport

$103(13.3)$

Number of bicycles available to use to get to school (n)

None

$215(28.1)$

One

$157(20.5)$

Two or more

$392(51.3)$

Number of vehicles at home (n)

None

$35(4.6)$

One

$263(34.4)$

Two or more

$466(61.0)$ 
Table 2. Transport to school in the previous two weeks and estimated time to walk and cycle to school

Total sample $(n=774)$

Walking to School Cycling to School

n (\%) n (\%)

Frequency of cycling to school in the

previous two weeks

Never

Almost never

Sometimes

Almost every day

Every day

Estimated time to walk/cycle to school

1-5 minutes

6-10 minutes

11-20 minutes

21-30 minutes

$31+$ minutes

Don't know
$211(27.6)$

$71(9.3)$

$110(14.4)$

149 (19.5)

$223(29.2)$

$84(11.0)$

$124(16.2)$

$187(24.5)$

$180(23.6)$

$172(22.5)$

$17(2.2)$
$714(93.5)$

$19(2.5)$

$16(2.1)$

$8(1.0)$

$7(0.9)$
$182(23.8)$

134 (17.5)

$134(17.5)$

$54(7.1)$

36 (4.7)

224 (29.3) 
Table 3. Attitudes, motivations, barriers and perceived behavioural control for walking and cycling to school

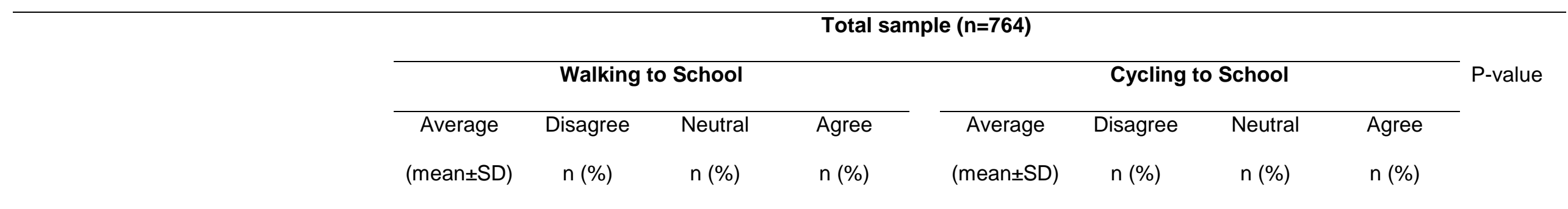

\section{Attitudes towards walking/cycling}

\section{to school}

\section{Experiential beliefs}

(Walking/cycling is interesting/

$$
4.3 \pm 1.4
$$$$
177(23.2)
$$

pleasant/ stimulating)

Instrumental beliefs

(Walking/cycling is healthy/

$$
5.4 \pm 1.4
$$

good/ useful)

\section{Subjective/perceived norm}

My parents or guardians think I

should walk/cycle to school†

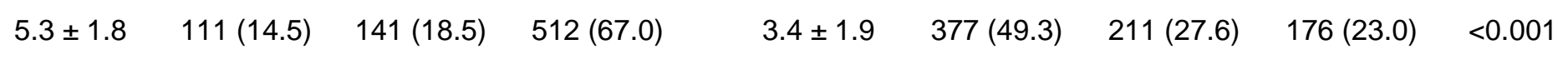


One or both of my parents or

guardians walk/cycle

frequently $\dagger$

My friends think I should

walk/cycle to school†

It is not considered cool to

walk/cycle to school*

No other students walk/cycle to

school†

Number of friends who always

or sometimes cycle to school $(0$ to 5)

My school encourages me to walk/cycle to school*

\section{Perceived behavioural control}

I am confident I could

walk/cycle to school†
$3.2 \pm 2.2 \quad 460(60.2) \quad 84(11.0) \quad 220(28.8)$

$1.9 \pm 1.8 \quad 642(84.0)$

$36(4.7)$

$86(11.3)$

$<0.001$

$370(48.4)$

$3.2 \pm 1.9 \quad 369(48.3)$

$251(32.9)$

$144(18.8)$

$<0.001$

$1.5 \pm 0.7 \quad 692(90.6)$

N/A

$72(9.4)$

$2.1 \pm 1.1 \quad 512(67.0)$

N/A

$252(33.0)$

$<0.001$

$1.8 \pm 0.9 \quad 594(77.7)$

N/A

170 (22.3)

$2.6 \pm 1.1 \quad 334(43.7)$

N/A

430 (56.3)

$<0.001$

$2.1 \pm 1.5$

N/A

N/A

$N / A$

$0.3 \pm 0.7$

$\mathrm{N} / \mathrm{A}$

N/A

$\mathrm{N} / \mathrm{A}$

$<0.001$

$2.0+0.9$

529 (69.2)

$0(0.0)$

235 (30.8)

$1.8 \pm 0.8 \quad 615(80.5)$

$0(0.0)$

149 (19.5)

$<0.001$

$5.9 \pm 1.7 \quad 82(10.7) \quad 64(8.4) \quad 618(80.9)$

$4.6 \pm 2.3 \quad 255(33.4)$

$74(9.7)$

435 (56.9)

$<0.001$ 
I have complete control over

whether or not I walk/cycle to

$$
5.1 \pm 1.9 \quad 155(20.3)
$$

$516(67.5)$

$5.4 \pm 2.0$

$134(17.5)$

$88(11.5)$

$542(70.9)$

$<0.001$

school†

\section{Behavioural intentions}

I want to regularly walk/cycle to

school†

$$
4.2 \pm 2.2
$$

$304(39.8)$

$122(16.0)$

$338(44.2)$

$1.9 \pm 1.5$

$658(86.1)$

$41(5.4)$

$65(8.5)$

$<0.001$

I intend to walk/cycle to school

$4.6 \pm 2$

$276(36.1)$

$77(10.1)$

$411(53.8)$

$1.5 \pm 1.3$

$693(90.7)$

$40(5.2)$

$31(4.1)$

$<0.001$

frequently†

*Data collected on a 4-point Likert scale (1=strongly disagree to $4=$ strongly agree). Data recoded as 1,2=disagree and 3,4=agree to create categorical variables.

†Data collected using a 7-point Likert scale ( $1=$ strongly disagree to $7=$ strongly agree). Data recoded as $1,2,3=$ disagree, $4=$ neutral and 5,6,7=agree to create categorical variables. 
Table 4. Personal incentives and barriers, environmental factors and safety perceptions of walking and cycling to school

Total sample $(n=764)$

\begin{tabular}{ccccccc}
\hline \multicolumn{3}{c}{ Walking to school } & & & \multicolumn{3}{c}{ Cycling to school } \\
\cline { 1 - 1 } \cline { 5 - 6 } Average & Disagree & Agree & & Average & Disagree & Agree \\
$($ mean \pm SD $)$ & $\mathrm{n}(\%)$ & $\mathrm{n}(\%)$ & & $($ mean \pm SD $)$ & $\mathrm{n}(\%)$ & $\mathrm{n}(\%)$
\end{tabular}

\section{Personal incentives}

Waking/cycling to school is a great way to get some exercise

I can chat to my friends on my walk/cycle to school

\section{Personal barriers}

Waking/cycling to school takes too much time

It involves too much planning ahead to

walk/cycle to school

I get too hot and sweaty walk/cycling to school

I have too much stuff to carry to walk/cycle to school

It is not convenient for me to walk/cycle to school because of my after-school schedule
$3.3 \pm 0.7$

$71(9.3)$

$693(90.7)$

$3.2 \pm 0.9$

$122(16.0)$

$642(84.0) \quad<0.001$

$2.4 \pm 1.1$

$401(52.5)$

$363(47.5)$

$1.6 \pm 0.8$

$670(87.7)$

$94(12.3)$

$<0.001$

$2.3 \pm 1.1$

$421(55.1)$

$343(44.9)$

$1.9 \pm 0.9$

$580(75.9)$

$184(24.1) \quad<0.001$

$1.6 \pm 0.9$

$640(83.8)$

$124(16.2)$

$2.1 \pm 1.0$

$491(64.3)$

$273(35.7) \quad<0.001$

$1.9 \pm 1.0$

$530(69.4)$

$234(30.6)$

$2.3 \pm 1.1$

$426(55.8)$

$338(44.2) \quad<0.001$

$2.4 \pm 1.1$

$382(50.0)$

$382(50.0)$

$2.8 \pm 1.1$

$282(36.9)$

$482(63.1)<0.001$

$2.0 \pm 1.0$

$519(67.9)$

$245(32.1)$

$1.4 \pm 1.1$

$409(53.5)$

$355(46.5) \quad<0.001$ 


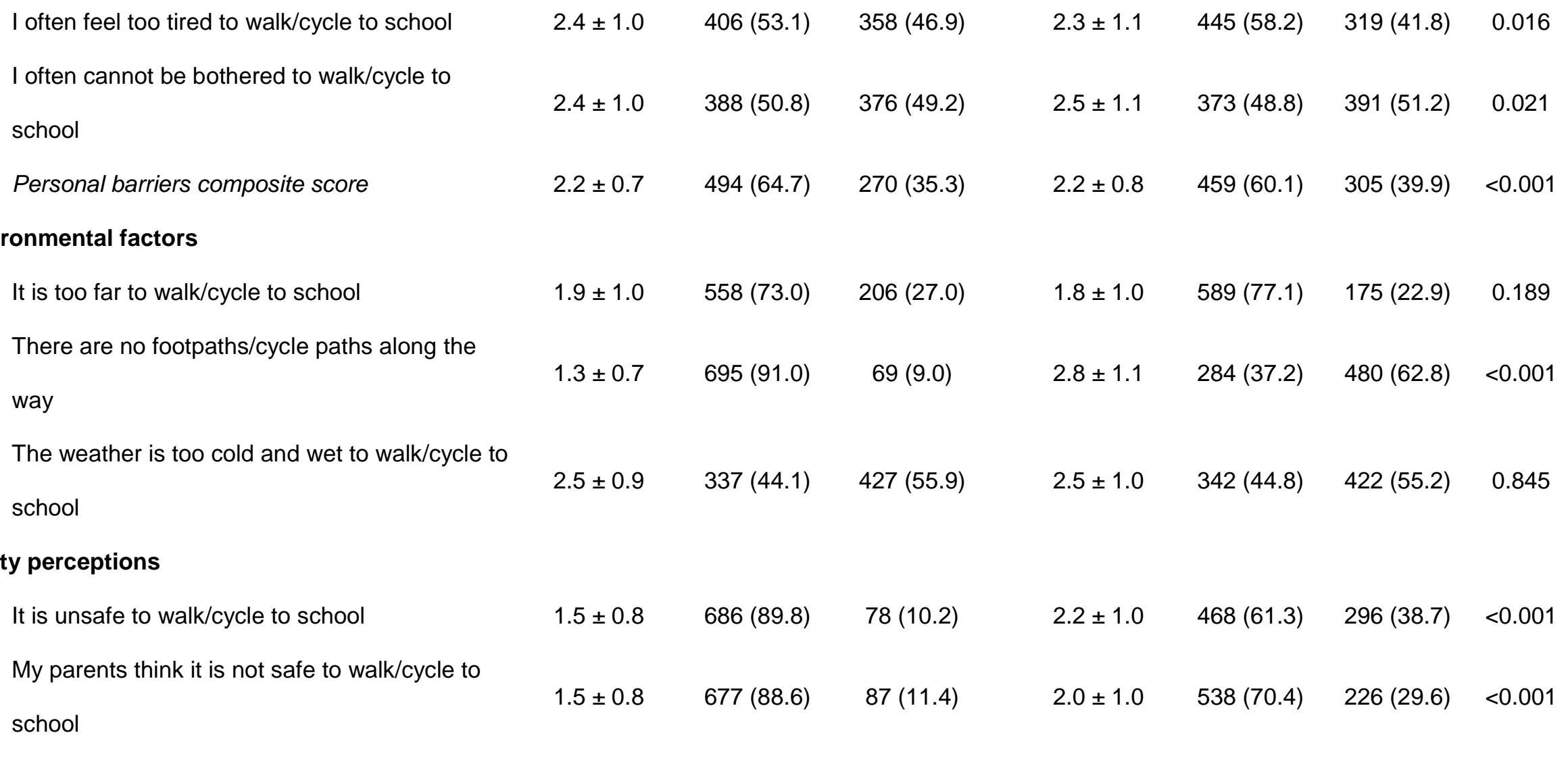

Data collected on a 4-point Likert scale (1=strongly disagree to 4=strongly agree). Data recoded as 1,2=disagree and 3,4=agree to create categorical variables. 


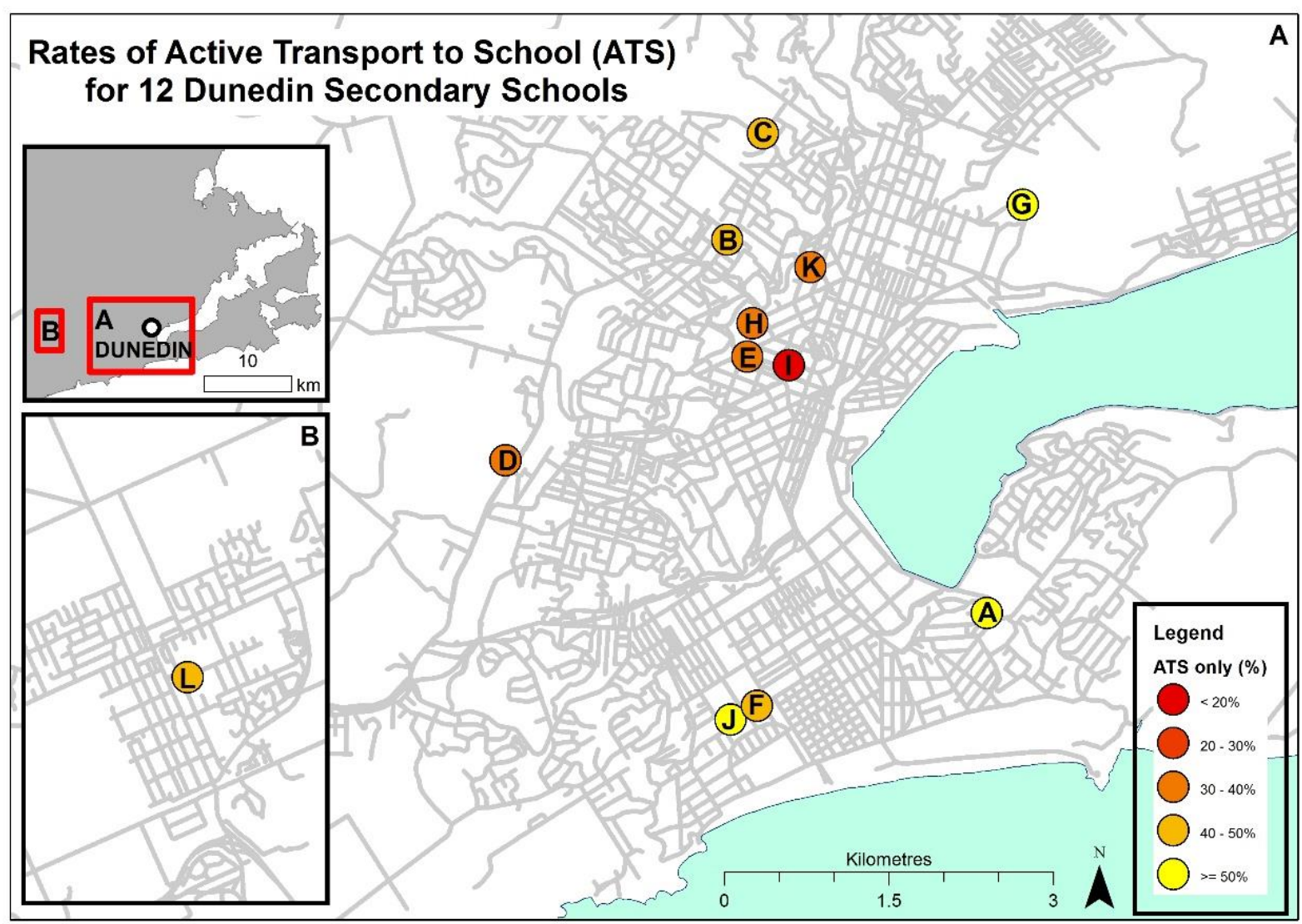

Figure 1. Rates of active transport to school in Dunedin secondary schools (ATS $=$ Active transport to school) 